\title{
Impact evaluation of conducted UWB transients on loads in power-line networks
}

\author{
Bing Li and Daniel Månsson \\ Department of Electromagnetic Engineering, KTH Royal Institute of Technology, Stockholm, Sweden \\ Correspondence to: Bing Li (libing@kth.se)
}

Received: 23 December 2016 - Revised: 11 April 2017 - Accepted: 10 June 2017 - Published: 21 September 2017

\begin{abstract}
Nowadays, faced with the ever-increasing dependence on diverse electronic devices and systems, the proliferation of potential electromagnetic interference (EMI) becomes a critical threat for reliable operation. A typical issue is the electronics working reliably in power-line networks when exposed to electromagnetic environment. In this paper, we consider a conducted ultra-wideband (UWB) disturbance, as an example of intentional electromagnetic interference (IEMI) source, and perform the impact evaluation at the loads in a network. With the aid of fast Fourier transform (FFT), the UWB transient is characterized in the frequency domain. Based on a modified BaumLiu-Tesche (BLT) method, the EMI received at the loads, with complex impedance, is computed. Through inverse FFT (IFFT), we obtain time-domain responses of the loads. To evaluate the impact on loads, we employ five common, but important quantifiers, i.e., time-domain peak, total signal energy, peak signal power, peak time rate of change and peak time integral of the pulse. Moreover, to perform a comprehensive analysis, we also investigate the effects of the attributes (capacitive, resistive, or inductive) of other loads connected to the network, the rise time and pulse width of the UWB transient, and the lengths of power lines. It is seen that, for the loads distributed in a network, the impact evaluation of IEMI should be based on the characteristics of the IEMI source, and the network features, such as load impedances, layout, and characteristics of cables.
\end{abstract}

\section{Introduction}

In modern society, the loads distributed in power-line networks are not limited to conventional electrical loads. With the development of power-line communication, smart grids, etc., more diverse loads characteristics appear. Modern electronics, for instance power-line modems and smart meters, can be directly connected to the power-line network. The integrated, digital, high-frequency operation trend makes these electronics more sensitive to electromagnetic (EM) disturbance, and correspondingly increases their vulnerability when the power-line network is exposed to EM environment (Meng et al., 2005; Vallbe et al., 2011).

Generally speaking, there are various types of EM signals, and the intentional electromagnetic interference (IEMI) is a particular class of high power EM threats to civilian society (Giri and Tesche, 2004; Månsson, 2008). The intentional generation of EM energy for terrorist or criminal purposes produces strong disturbance, thus interfering with or even damaging the critical electronic systems (Radasky et al., 2004). Nowadays, the increased dependence on diverse electronic devices and systems, as well as the proliferation of high power EM sources, emphasize the great importance of studying the behavior of IEMI and its consequences.

Numerous efforts have been devoted to the investigation of IEMI and its impact. In Månsson et al. (2009), the IEMI-cube is proposed for IEMI vulnerability evaluation, which implies that, it is helpful to have a comprehensive impact evaluation. The vulnerability of different kinds of systems in engineering applications, against IEMI, is extensively investigated in Hoad et al. (2004), Månsson et al. (2008b), Bayram et al. (2008) and Beek and Leferink (2015).

According to the classification of IEMI source waveforms presented in Månsson (2008), the ultra-wideband (UWB) source is a typical high-power electromagnetic (HPEM) signal, which produces a very fast time-domain transient (however any waveform could be used in the investigation). Although the energy of the UWB transient is limited due to the very short duration, the resulting interference may still 
be significant. Many studies regarding the UWB transient waveform itself and its potential impacts in different scenarios have been widely conducted in recent years, and related results can, e.g., be found in Camp et al. (2004); Camp and Garbe (2004) and Månsson et al. (2007, 2008a).

During the past decade, the feasibility of applying the transmission-line (TL) theory to UWB sources was always a hot topic that has drawn much attention and been extensively discussed, e.g., in Rachidi and Tkachenko (2008). In Weber and ter Haseborg (2004), several techniques for measuring the conducted HPEM signals in the TL scenario were proposed. In Månsson et al. (2008a), the propagation issue of the UWB transient in low-voltage power network of a building was discussed, where several measures from the perspective of installation, in the light of experimental results, were suggested to reduce the risk of conducted IEMI. Recent studies regarding IEMI in electrical networks are presented in Li et al. (2015); Li and Månsson (2016) and Li and Månsson (2015), where propagation models of EM disturbances were established based on the TL theory and the Baum-Liu-Tesche (BLT) approach proposed by Baum et al. (1978); Baum (1995). Generally speaking, the method is applied in the frequency domain. Recently, the BLT equation in the time domain is developed and applied to a coaxial cable (Tesche, 2007). However, it should be noted that, when applying the time-domain method, there is a huge challenge, namely, the tractability of computation. More precisely, considering the complexity, the time-domain analysis presented in Tesche (2007) only applies to a simple network structure, while it is not feasible when the network gets more complex, e.g., with multiple junctions or branches. Due to this fact, we therefore seek a solution in the frequency domain to deal with complex networks, rather than in the time domain.

In this paper, we consider a multi-junction power-line network, and the objective of our research is to investigate the impact by an IEMI source on a targeted load. The load impedance is complex and a series of impact evaluation quantifiers are used. Regarding the IEMI source, we in particular model a conducted UWB transient (Sabath and Mokole, 2014) as a double exponential pulse, where the rise time and pulse width vary, thereby producing a significant effect on some of the quantifiers in the time domain. Furthermore, the factors, such as the structure of the power-line network (e.g., length of power line) and the attribute of the loads (complex impedance) connected to the network, are also taken into consideration for our investigation.

The rest of the paper is organized as follows. In Sect. 2, we outline the research methodology, which is followed by related techniques. Specifically, we describe the computation method of load responses in complex networks, and the criteria for impact evaluation. In Sect. 3, we give the analytical model, including the description of the injected UWB transient and network model, and we also briefly present the mathematical methods to be used. In Sect. 4, we calculate the transient response of the observed load, and obtain results in

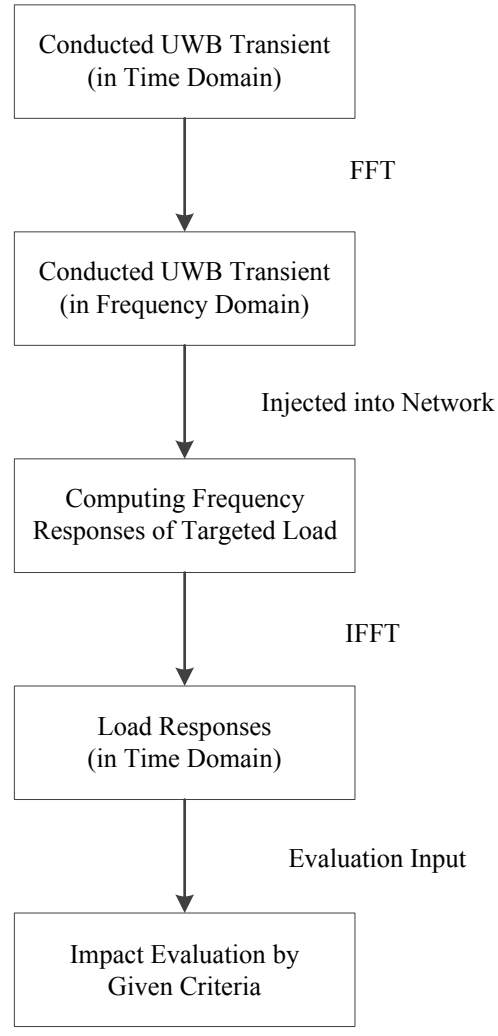

Figure 1. Diagram of research methodology.

terms of the five quantifiers. Moreover, we discuss the effects of different load impedances (resistive, capacitive, or inductive) connected to the network, different rise times and pulse widths of the UWB transient, and different lengths of the power lines. Finally, we conclude the work.

\section{Methodology and related techniques}

\subsection{Methodology}

In this work, we consider the impact of a conducted UWB transient on loads in power-line networks. The UWB transient is differentially injected into the power line, and transverse electromagnetic (TEM) or quasi-TEM is the main mode of wave propagation. The methodology of our study, as outlined in Fig. 1, consists of the following four phases:

1. Regarding the time-domain conducted UWB transient, we apply the fast Fourier transform (FFT) to convert the disturbance signal to its corresponding frequencydomain representation.

2. As IEMI source, the converted disturbance signal is injected into the objective power-line network in the frequency domain. Through computations of the load responses, the influence, characterized by frequency responses, can be obtained. 
3. Then, we accordingly employ the inverse FFT (IFFT) to convert from the outputted frequency responses to the time-domain load responses.

4. Finally, we evaluate the impact of conducted UWB transient in the light of achieved time-domain data, according to the evaluation criteria of interest.

Clearly, there are two issues playing critical roles during the evaluation, i.e., computation of frequency responses, and the impact evaluation criteria. In what follows, we will provide more related details for above two issues.

\subsection{Technical descriptions}

\subsubsection{Computation of frequency response}

To compute the frequency response of loads in networks, there are mainly two categories of means: (i) commercial simulators with graphical user interface (here, we have used the EMEC software (Carlsson et al., 2004)), and (ii) analytical computations (Baum et al., 1978; Baum, 1995; Anatory et al., 2009; Shin et al., 2011; Li et al., 2015). Considering the diversity of power-line networks, we in this study focus on the method of analytical computations, since the implementation as well as the adjustment of a broad range of network parameters can often be more easily and flexibly manipulated, compared with commercial software.

Despite that numerous achievements on the analytical approach of computing the frequency responses in power-line networks have been obtained, there are still application limitations when dealing with complex networks in terms of the topology. To be more precise, in the presence of multiple junctions, as shown in Fig. 2, the emergence of multi-path propagation, resulted by the multiple reflections between junctions, hinders the utilization of those approaches based on the conventional BLT equation (Baum et al., 1978; Baum, 1995). On the other hand, even though the problem can be addressed by capturing the global characteristics of the entire power-line network, a very high computational complexity will also be generated when the network topology grows complex (Anatory et al., 2009; Shin et al., 2011).

In order to deal with various complex networks, and maintain low computational complexity, we here applied an efficient analytical computation method that was proposed in Li et al. (2015). This is based on modified BLT equations and implemented in a recursive manner. By this approach, the entire original network is first decomposed into multiple equivalent sub-networks, then only the local network information is required for iterative calculation and parameter updates (see Sect. 3.2 for description).

\subsubsection{Criteria for impact evaluation}

To perform the impact evaluation, we use several criteria. Reference (Taylor and Giri, 1994) proposed five common,

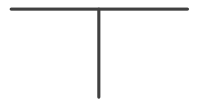

(a)

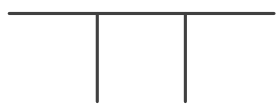

(c)

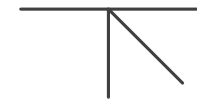

(b)

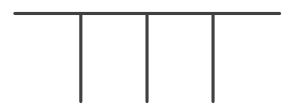

(d)
Figure 2. Illustration of power-line networks with multiple junctions.

but important quantifiers (often also called "Norms"), which differ in nature, for example dielectric breakdown, heating effects, etc. Following from the metrics introduced in Taylor and Giri (1994), in our study we use the following five criteria to assess the influence of conducted UWB transient on loads, here $x(t)$ represents the load voltage:

a. time-domain peak

$$
Q_{1} \triangleq \sup _{0<t<\infty}|x(t)|
$$

b. total signal energy

$$
Q_{2} \triangleq \int_{0}^{\infty}|x(t)|^{2} \mathrm{~d} t ;
$$

c. peak signal power

$$
Q_{3} \triangleq \sup _{0<t<\infty}|x(t)|^{2}
$$

d. peak time rate of change

$$
Q_{4} \triangleq \sup _{0<t<\infty}\left|\frac{\mathrm{d}}{\mathrm{d} t} x(t)\right|
$$

e. peak time integral of the pulse

$$
Q_{5} \triangleq \sup _{0<t<\infty} \int_{0}^{t} x(t) \mathrm{d} t .
$$

\section{Models and parameters}

\subsection{Double exponential UWB transient}

We consider a double exponential UWB transient as IEMI source, and the signal is expressed by

$a(t)=A_{0}\left(e^{-\alpha t}-e^{-\beta t}\right) \cdot u(t)$, 
Table 1. FFT-related values.

\begin{tabular}{ccccc}
\hline Parameter & $F_{\max }$ & $F_{\mathrm{S}}$ & $T$ & NFFT \\
\hline Value & $50 \mathrm{GHz}$ & $100 \mathrm{GHz}$ & $1 \times 10^{-11} \mathrm{~s}$ & 1024 \\
\hline
\end{tabular}

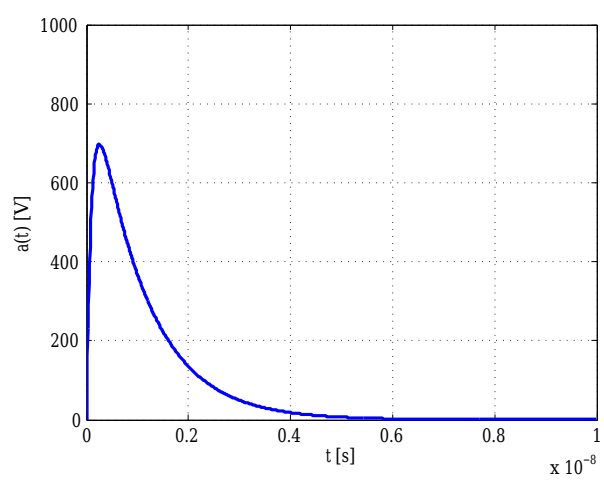

Figure 3. The time-domain waveform of the UWB transient, for $A_{0}=1 \mathrm{kV}, \alpha=10^{9}$ and $\beta=10^{10}$.

where $A_{0}$ is the amplitude amplifier, noting that $A_{0}=A_{0}(\alpha$, $\beta) . \alpha$ and $\beta$ relate to the full-width-at-half-maximum time $t_{\mathrm{fwhm}}$ and rise time $t_{\mathrm{r}}$ (10 to $90 \%$ of peak), respectively. $u(t)$ is the step function, to ensure $a(t) \equiv 0$, at $t<0$. In this paper, as an example, the time-domain waveform by Eq. (1), with respect to the parameters $A_{0}=1 \mathrm{kV}, \alpha=10^{9}$ and $\beta=10^{10}$, is illustrated in Fig. 3, for the following calculations and analyses.

To enable the subsequent analysis in the frequency domain, we apply FFT to capture the features of the UWB transient in the frequency domain. It is worth noting that, the pulse is a windowed signal, where the maximum frequency $F_{\max }$ is infinite, such that the Nyquist-Shannon sampling theorem cannot be applied for the lossless sampling. To ensure the accuracy, we count $F_{\max }$ when the frequencydomain magnitude is below the threshold, i.e., $\leq 1 \%$ of the maximum, and the sampling frequency $F_{\mathrm{S}}$ needs to satisfy $F_{\mathrm{s}} \geq 2 F_{\max }$. Please note that, although the maximum frequency is $50 \mathrm{GHz}$, according to the spectrum, the energy of the signal is mostly at less than $1 \mathrm{GHz}$. Here, we denote the sampling interval and number of sampling points by $T$ and NFFT, respectively. With the FFT-related values shown in Table 1, it can be verified that NFFT $=1024$ is able to provide sufficiently high accuracy to characterize the signal in the frequency domain.

\subsection{Power-line network}

In this paper, a specific network consisting of two junctions and four branches is investigated (see Fig. 4). The UWB transient is injected into the network from one port, and other three ports are connected to loads. For simplicity, load 1 and load 2 are assumed to be resistive, and load 3, with complex

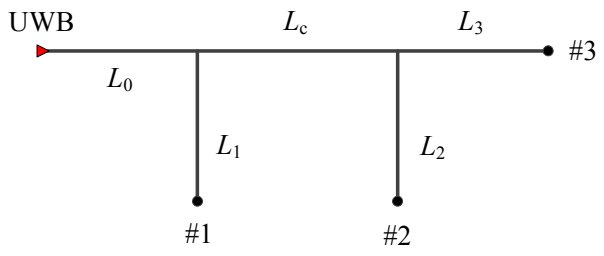

Figure 4. Example of a power-line network.

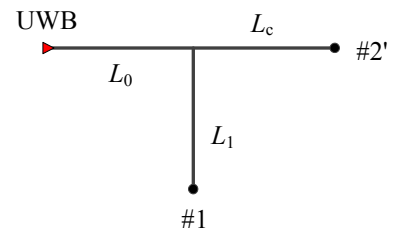

(a)

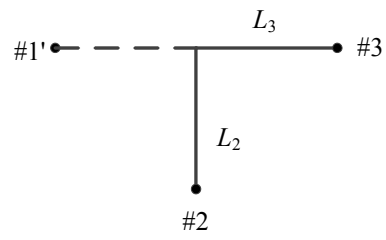

(b)
Figure 5. Decomposition of the network shown in Fig. 4.

impedance, varies in both amplitude and phase (of course, any load arrangement can be made to suit a specific situation better). Here we assume that Load 3 is time-independent and not dependent on the amplitude of signal in it. Regarding load $3\left(Z_{3}=R_{3} \pm j X_{3}=\left|Z_{3}\right| \angle \phi_{3}\right)$, the amplitude (i.e., $\left|Z_{3}\right|$ ) and phase (i.e., $\phi_{3}$ ) were here chosen from 1 to $1000 \Omega$ and from $-\pi / 2$ to $\pi / 2$, respectively. In this paper, we focus on the response of load 3, which is denoted as the "targeted load". Moreover, we initially assume that all power lines have the identical length, i.e., $L_{0}=L_{1}=L_{2}=L_{3}=L_{\mathrm{c}}$, and an identical characteristic parameter $Z_{\mathrm{c}}$ is applied for all branches (e.g., same type of cable). Parameters of the network are summarized in Table 2.

We use our introduced method ( $\mathrm{Li}$ et al., 2015), which is efficient and derived based on the BLT equation (Baum et al., 1978; Baum, 1995), to solve the frequency response of the load in the power-line network. The basic idea of the method is dividing a multi-junction network into several single junction networks, as shown in Fig. 5, and the cutting (dividing) point is at the second junction, which is connected with line $L_{\mathrm{c}}, L_{2}$ and $L_{3}$. Moreover, we introduce a virtual branch with zero length to preserve the characteristics of the single junction network, as shown in Fig. 5b.

For the one-junction network shown in Fig. 5a, the modified BLT equation is (see Li et al., 2015)

$\boldsymbol{R}=(\mathbf{I}+\mathbf{P})(\mathbf{I}-\boldsymbol{\Gamma} \mathbf{P})^{-1} \boldsymbol{S}$,

where $\boldsymbol{R}=\left[V_{0}, V_{1}, V_{2^{\prime}}\right]^{T}$ is the voltage vector showing the responses of the three loads. $\mathbf{I}$ is the identity matrix of size 3. $\mathbf{P}=\operatorname{diag}\left(\rho_{0}, \rho_{1}, \rho_{2^{\prime}}\right)$ is the reflection matrix, where $\rho_{i}=\left(Z_{i}-Z_{\mathrm{c}}\right) /\left(Z_{i}+Z_{\mathrm{c}}\right),(i=0,1)$ denote reflection coefficients at the two terminals of line $L_{0}$ and $L_{1}$, and $\rho_{2^{\prime}}$ is the corrected reflection coefficient at the second junction, which is calculated by Eq. (3). The transmission matrix $\Gamma$ and excitation source vector $S$ are respectively given by 
Table 2. Parameters in the model.

\begin{tabular}{ll}
\hline Parameter & Value \\
\hline$Z_{1}, Z_{2}$ & $100 \Omega$ \\
$\left|Z_{3}\right|$ & $1-1000 \Omega$ \\
$\phi_{3}$ & $-\pi / 2-\pi / 2$ \\
$Z_{\mathrm{c}}$ & $50 \Omega$ \\
$L_{\mathrm{c}}, L_{0}, L_{1}, L_{2}, L_{3}$ & $30 \mathrm{~m}$ \\
\hline
\end{tabular}

$\boldsymbol{\Gamma}=\left[\begin{array}{ccc}\rho_{1}^{(0)} e^{-2 \gamma L_{0}} & T_{1}^{(1)} e^{-\gamma\left(L_{0}+L_{1}\right)} & T_{1}^{(c)} e^{-\gamma\left(L_{0}+L_{\mathrm{c}}\right)} \\ T_{1}^{(0)} e^{-\gamma\left(L_{0}+L_{1}\right)} & \rho_{1}^{(1)} e^{-2 \gamma L_{1}} & T_{1}^{(c)} e^{-\gamma\left(L_{1}+L_{\mathrm{c}}\right)} \\ T_{1}^{(0)} e^{-\gamma\left(L_{0}+L_{\mathrm{c}}\right)} & T_{1}^{(1)} e^{-\gamma\left(L_{1}+L_{\mathrm{c}}\right)} & \rho_{1}^{(c)} e^{-2 \gamma L_{\mathrm{c}}}\end{array}\right]$, $\boldsymbol{S}=\left[\begin{array}{c}-\frac{1}{2}\left(V_{\mathrm{s}}-Z_{\mathrm{c}} I_{\mathrm{S}}\right)+\rho_{1}^{(0)} \cdot \frac{1}{2}\left(V_{\mathrm{s}}+Z_{\mathrm{c}} I_{\mathrm{S}}\right) e^{-\gamma L_{0}} \\ T_{1}^{(0)} \cdot \frac{1}{2}\left(V_{\mathrm{s}}+Z_{\mathrm{c}} I_{\mathrm{s}}\right) e^{-\gamma\left(L_{0}+L_{1}\right)} \\ T_{1}^{(0)} \cdot \frac{1}{2}\left(V_{\mathrm{s}}+Z_{\mathrm{c}} I_{\mathrm{s}}\right) e^{-\gamma\left(L_{0}+L_{\mathrm{c}}\right)}\end{array}\right]$,

where $\rho_{1}^{(j)}$ and $T_{1}^{(j)}$ are the reflection coefficient and transmission coefficient, respectively, for the first junction in Fig. 5a. The subscript is the index of the junction, and the superscript is the index of the branch, $j=0,1, c . V_{\mathrm{s}}$ and $I_{\mathrm{s}}$ represents the voltage source and current source, respectively. $\gamma$ denotes the propagation constant.

For a junction with $N+1$ branches, the reflection coefficient $\rho_{1}^{(j)}$ and transmission coefficient $T_{1}^{(j)}$ are respectively given by Månsson et al. (2008a)

$\rho^{(j)}=\frac{1-N}{1+N}$ and $T^{(j)}=1+\rho^{(j)}$.

Also, to calculate the load responses, we need to correct the reflection coefficient at the second junction, by using the following equations (see Li et al., 2015)

$\rho_{2^{\prime}}=T_{2}^{(c)}\left(1-\frac{T_{2}^{(2)}}{A}-\frac{T_{2}^{(3)}}{B}\right)^{-1}-1$,

with

$A=1+\frac{1}{\rho_{2} e^{-2 \gamma L_{2}}}, B=1+\frac{1}{\rho_{3} e^{-2 \gamma L_{3}}}$.

Similarly, by using the modified BLT equation described above, we calculate the voltage responses of the subnetwork shown in Fig. 5b, and obtain the result for the targeted load.

It is worth noting that the solved voltage vector $\boldsymbol{R}$ is presented in the frequency domain. Since FFT has been applied to transfer the UWB transient to the frequency domain, the time-domain responses of the loads can be obtained via IFFT.

\section{Results and analyses}

\subsection{General results by five quantifiers}

With the aforementioned analytical model, the results of load 3 (i.e., $Z_{3}$ ) are obtained, in terms of the five quantifiers.
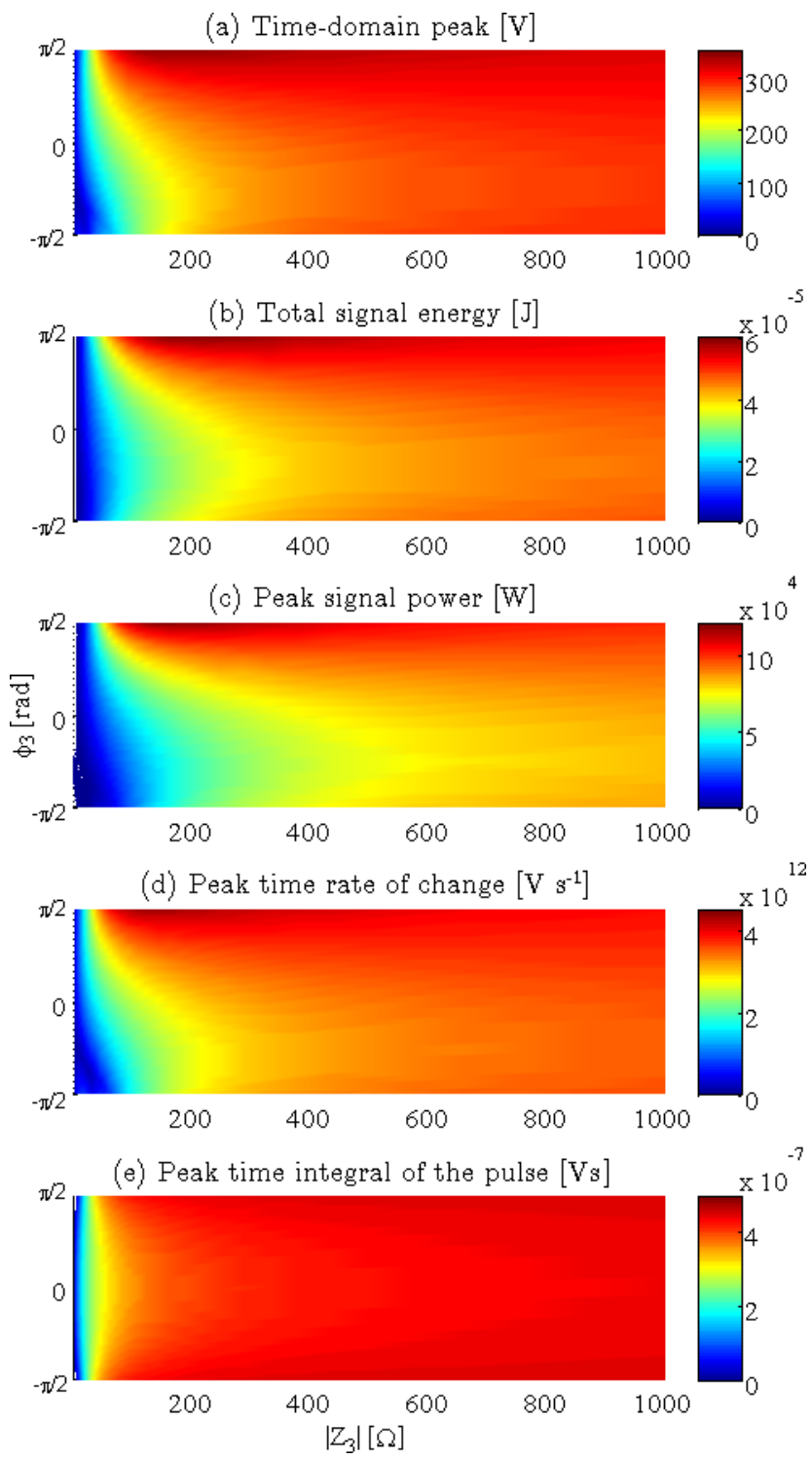

Figure 6. Calculation results of five quantifiers. (In the form of $\left|Z_{3}\right| \angle \phi_{3}$.)

For the polar coordinate expression, $Z_{3}=\left|Z_{3}\right| \angle \phi_{3}$, we can see in Fig. 6 that:

1. Basically, for Fig. 6a to d, they all have same form. Obvious changes happen in the region of low amplitudes (here $\left|Z_{3}\right| \leqslant 300 \Omega$ ). With the amplitude increasing, the rate of change is different at different phases. From inductive to capacitive, the value first decreases and then increases at certain amplitude, and the highest value happens at purely inductive. Particularly, one peak emerges at dominantly inductive behavior. It implies that dominantly inductive load, with certain amplitudes, may suffer the most from conducted UWB transients. However, in the situation that load 3 mainly exhibits resistive (i.e., $\phi_{3} \approx 0$ ) or dominantly capacitive features , 
the impacts of IEMI on these quantifiers do not change significantly.

2. In Fig. 6e, for all phase values, the quantifier increases monotonically with the growing amplitude. The increase is, more or less, independent of the phase value.

3. The time-domain peak (Fig. 6a) and peak signal power (Fig. 6c) have similar behavior. It is due to the fact that they get linked to each other through a deterministic relation, namely, via the square of the parameter of the quantifiers.

In addition, we provide another perspective, i.e., $Z_{3}=R_{3} \pm j X_{3}$, to investigate the results via the five quantifiers, as shown in Fig. 7. It can be seen that the maximum values of the first four quantifiers are concentrated at $R_{3}=0$, and $X_{3} \leqslant 300 \Omega$, i.e., load 3 is purely inductive. For peak time integral of the pulse (Fig. 7e), the results increase with amplitude, and along the direction of the real axis, which represents the resistance of load, the gradient is smaller than other directions. Considering the mapping $R_{3} \pm j X_{3}=\left|Z_{3}\right| \angle \phi_{3}$, the observations in the polar coordinate expression are in line with what are shown in the $Z_{3}=R_{3} \pm j X_{3}$ format.

Based on the above analysis, we can conclude that, from the perspective of minimizing the impact of IEMI, it will be a wise choice to increase the resistive attribute of the targeted load and to decrease the amplitude $\left(\left|Z_{3}\right|\right)$. Moreover, it should be noted that the attribute of load (capacitive, resistive, or inductive) is an important factor causing a high peak, in terms of the first four quantifiers. Also, since some quantifiers are related to each other, the IEMI-induced impacts are similar (refer to Fig. 6a and c).

\subsection{Effect of load impedance}

In this subsection, we study the effect of load impedance. To implement this investigation, we sequentially set load 1 , load 2 or load 3 , to be complex (including resistive, capacitive, or inductive parts), and fix the other two to be $100 \Omega$ resistive loads. For the targeted load 3 , the overall trends of the five quantifiers, in the three cases studied here, are similar to the results we got in Fig. 6, however, with different maximum values. Therefore, we mainly focus on the maximum values that the targeted load may suffer, in each group of figures (here we have three groups).

The maximum values of the five quantifiers, regarding load 3, are shown in Fig. 8. We can find that, the values of the first four quantifiers, when load 3 is complex, are higher in general, while the value of the last quantifier is constant. That means, the targeted load, in terms of quantifiers, gets more affected when the variation of amplitude and phase happens to itself, rather than to other loads in the network.
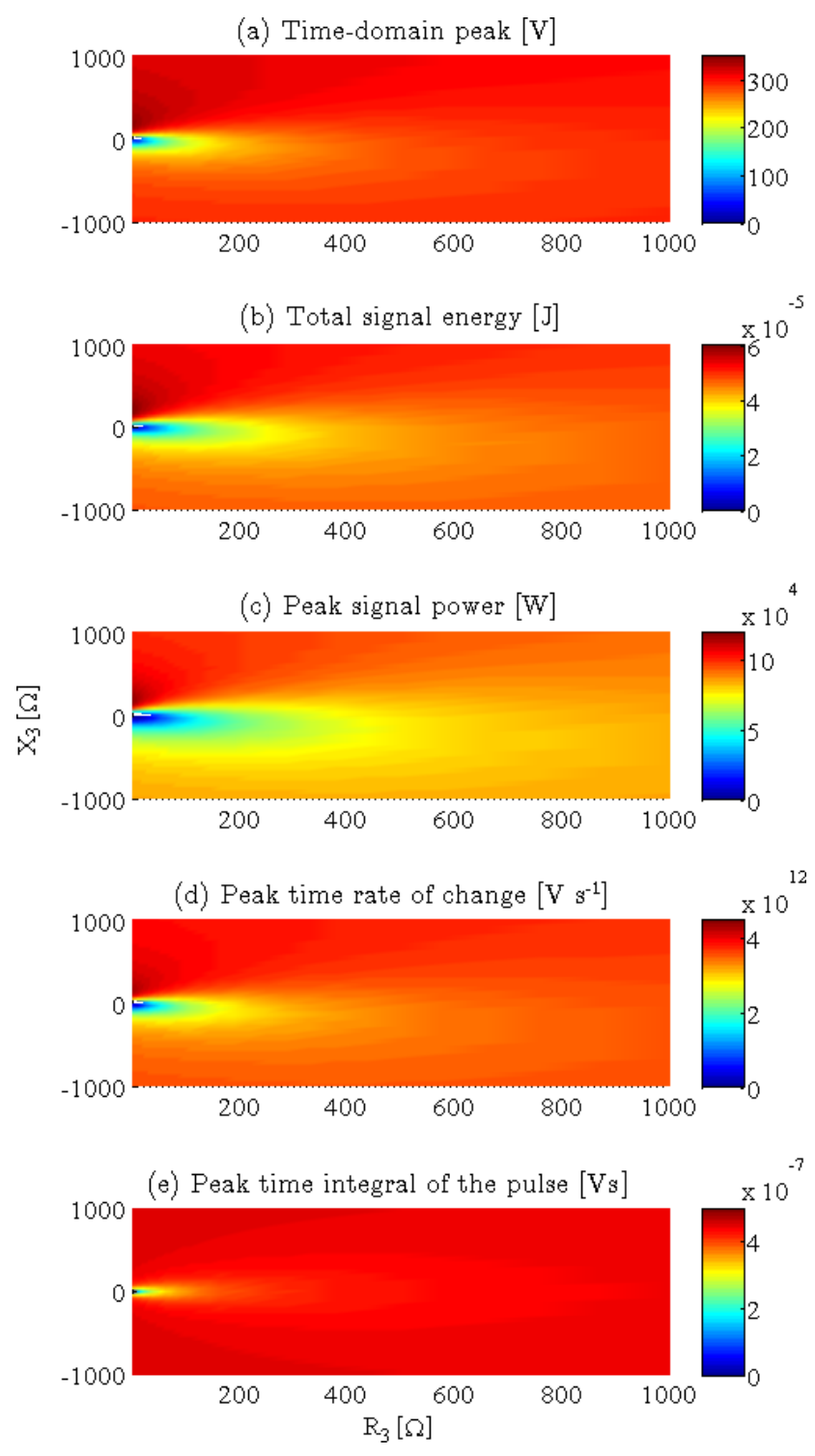

Figure 7. Calculation results of five quantifiers. (In the form of $\left.R_{3} \pm j X_{3}\right)$.

\subsection{Effect of $\alpha$ and $\beta$}

Regarding the expression of the double exponential UWB transient as shown in Eq. (1), the specific IEMI source is characterized by three important parameters, i.e., $A_{0}, \alpha$ and $\beta$, which are related to rise time $t_{\mathrm{r}}$ and pulse length $t_{\mathrm{fwhm}}$. To investigate the impact of UWB transients, it is necessary to analyse the effects when UWB pulses (with different rise times and pulse widths) are applied at the injection port. One efficient method to investigate this is to investigate the dependency upon the ratio of $\beta / \alpha$ (Mao and Zhou, 2008; Camp and Garbe, 2004), which can be implemented by keeping $A_{0}$ and $\alpha$ constant and varying $\beta$. Change the ratio from 2 to 500 , 


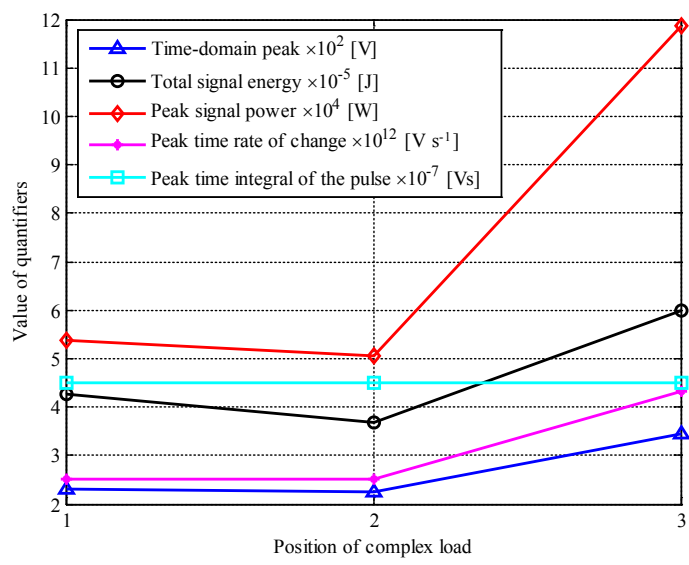

Figure 8. The maximum values of the five quantifiers, when changing the position of the complex load.

and some of the corresponding time-domain waveforms are shown in Fig. 9.

Regarding the targeted load 3, the overall trends of the five quantifiers, under different $\beta / \alpha$ ratios, are still similar to the results shown in Fig. 6. Thus, we only focus on the maximum values of the five quantifiers (see Fig. 10). In this group of curves, generally speaking, the impacts on the five quantifiers increase, when $\beta / \alpha$ grows.

Specifically, with the increase of $\beta / \alpha$, i.e., the rise time of the pulse gets shorter, we can observe that, time-domain peak (blue curve), total signal energy (black curve) and peak time integral of the pulse (cyan curve) have similar trends, while peak signal power (red curve) and peak time rate of change (pink curve) have similar trends. When $2 \leqslant \beta / \alpha \leqslant 100$, both of the two groups of curves have a rapid growth rate. When $\beta / \alpha$ continues increasing to 500 , all of the growth trends slow down, and the growth rate of the first group becomes much slower than the latter (even stops increasing).

Therefore (as could be expected), we can conclude that, for UWB transients, $\beta / \alpha$ plays an important role in affecting the quantifiers' behavior. As the ratio increases, the impact, that the UWB transient produced, on the targeted load keeps increasing, although the growth rate decays (not in the same way).

\subsection{Effect of the lengths of power lines}

It is revealed in our previous work (Li and Månsson, 2017) that, the lengths of the power lines affect load responses. In this subsection, the focus is on the effect from varying the lengths of the power lines. Here, we take the scenario in which each line has an identical length, i.e., $L_{\mathrm{c}}=L_{0}=L_{1}=L_{2}=L_{3}=30 \mathrm{~m}$, as a reference. First, we investigate the effect by varying the lengths of power lines together, i.e., making $L_{\mathrm{c}}=L_{0}=L_{1}=L_{2}=L_{3}=3,10$ or $100 \mathrm{~m}$, and compare the results (given in Fig. 11) with the result of the reference scenario (see Fig. 6). In each case, as

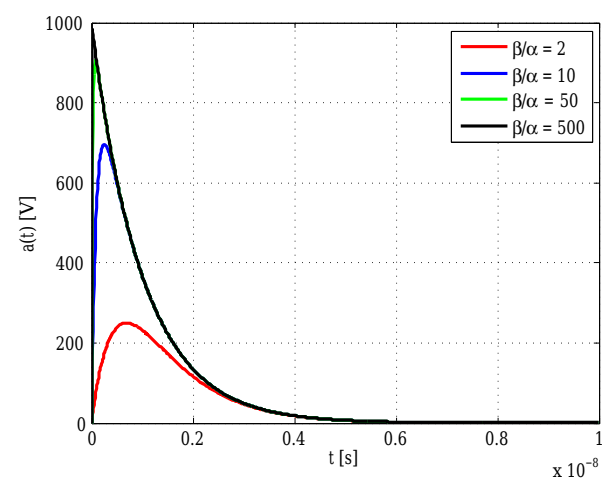

Figure 9. Different waveforms of the UWB transients in the time domain.

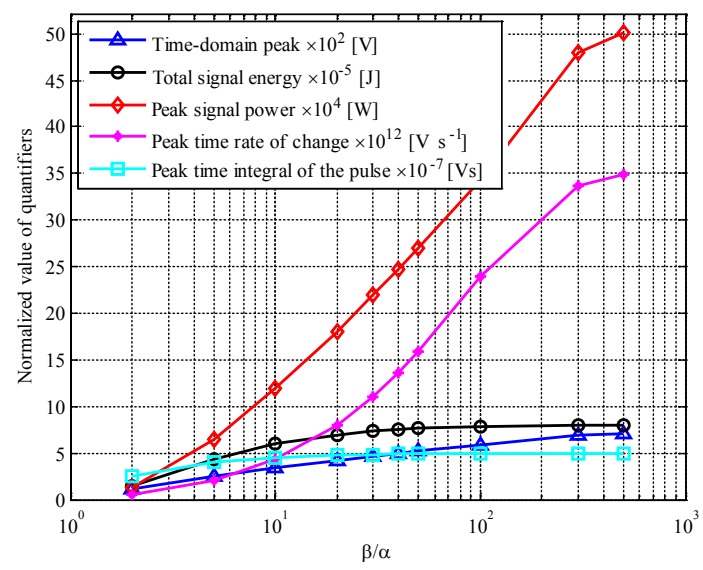

Figure 10. The maximum values of the five quantifiers, when changing the ratio $\beta / \alpha$.

shown in Fig. 11, the overall trends of the five quantifiers are different from the trends we observed in Fig. 6 . When the lengths are $3 \mathrm{~m}$, two peaks emerge at $\phi_{3}=-\pi / 2$ and $\pi / 2$, respectively, when considering the first four quantifiers. Taking the phase $\phi=0$ as the dividing line, it is evident that for time-domain peak and peak signal power, the region of peak in the upper half-plane ( $\phi>0$, corresponding to the inductive region) is obviously larger than the peak in the capacitive region $(\phi>0)$, which shows that the inductive targeted load will suffer more impact, in terms of these two quantifiers. However, total signal energy and peak time rate of change behave in the opposite manner. At high amplitudes, the values of these four quantifiers are much lower than the ones in Fig. 6. When the lengths are $10 \mathrm{~m}$, the values of quantifiers increase monotonically with the growing amplitude at each phase, thus having no peaks (in contrast to that for the case with 3 or $30 \mathrm{~m}$ ). When the lengths are $100 \mathrm{~m}$, the overall trends are opposite to what we found in Fig. 6, i.e., the peaks emerge at dominantly capacitive behavior and the highest value happens at purely capacitive impedance. 


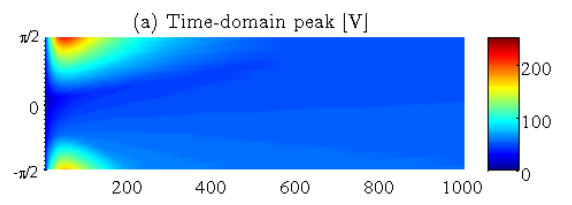

(b) Total signal energy [J]

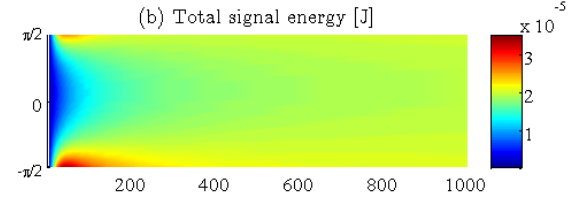

(c) Peak signal power [W]

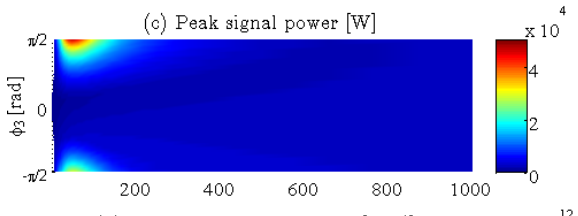

(d) Peak time rate of change $\left[\mathrm{V} \mathrm{s}^{-1}\right]$
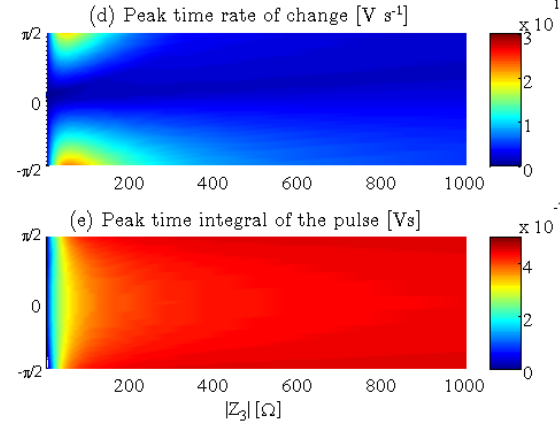

(a) $3 \mathrm{~m}$

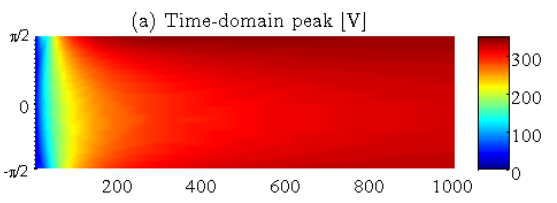

(b) Total signal energy [J]

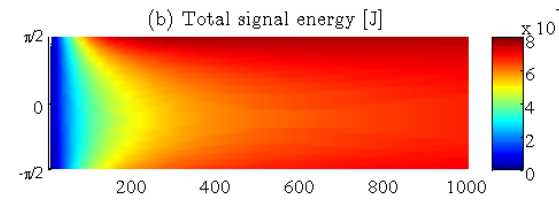

(c) Peak signal power [W]

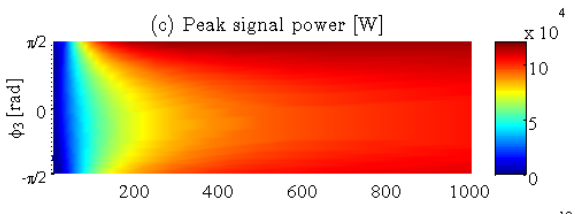

(d) Peak time rate of change $\left[\mathrm{V} \mathrm{s}^{-1}\right]$
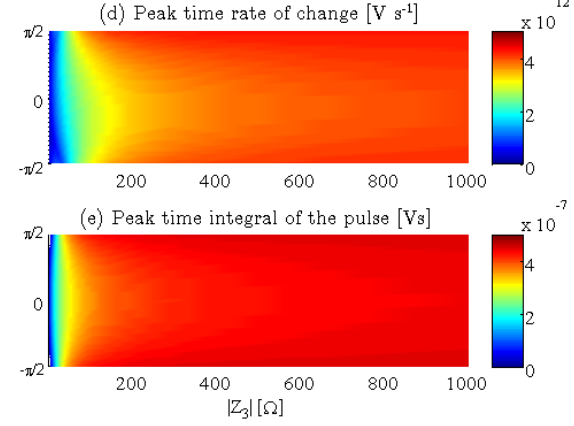

(b) $10 \mathrm{~m}$

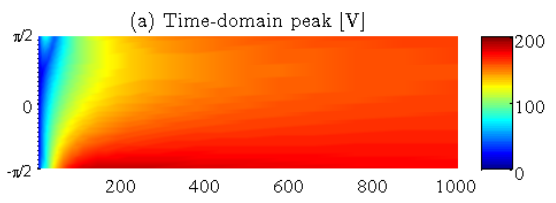

(b) Total signal energy [J]

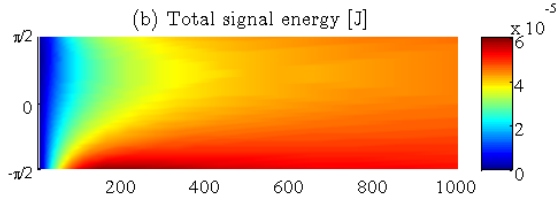

(c) Peak signal power [W]
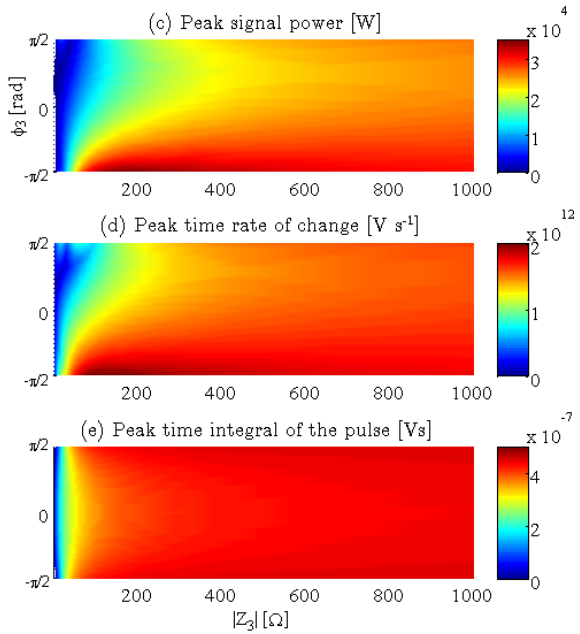

(c) $100 \mathrm{~m}$

Figure 11. Results of five quantifiers, when the lengths of power lines are 3,10 , or $100 \mathrm{~m}$. (In the form of $\left|Z_{3}\right| \angle \phi_{3}$.)

Table 3. Different cases studied.

\begin{tabular}{ll}
\hline Case number & Line doubled \\
\hline 1 & Reference \\
2 & $L_{\mathrm{c}}$ \\
3 & $L_{0}$ \\
4 & $L_{1}$ \\
5 & $L_{2}$ \\
6 & $L_{3}$ \\
\hline
\end{tabular}

Next, for the cases to be investigated, we sequentially double the length of only one of the lines each time, while the rest remain to be $30 \mathrm{~m}$, respectively. In this case, we need to investigate six cases altogether, as given in Table 3. Likewise, as what we did in Sects. 4.2 and 4.3, we plot the maximum values of the five quantifiers in Fig. 12, due to the overall trends of the quantifiers in the studied cases are similar to the trends shown in Fig. 6. Generally, total signal energy (black curve) and peak signal power (red curve) in Fig. 12 change obviously, while other three quantifiers do not. Specifically, the values of black and red curves decrease at Case 3 and Case 6, and increase at Case 2, Case 4 and Case 5, which indicates that increasing only $L_{0}$ or $L_{3}$ helps reduce the im-

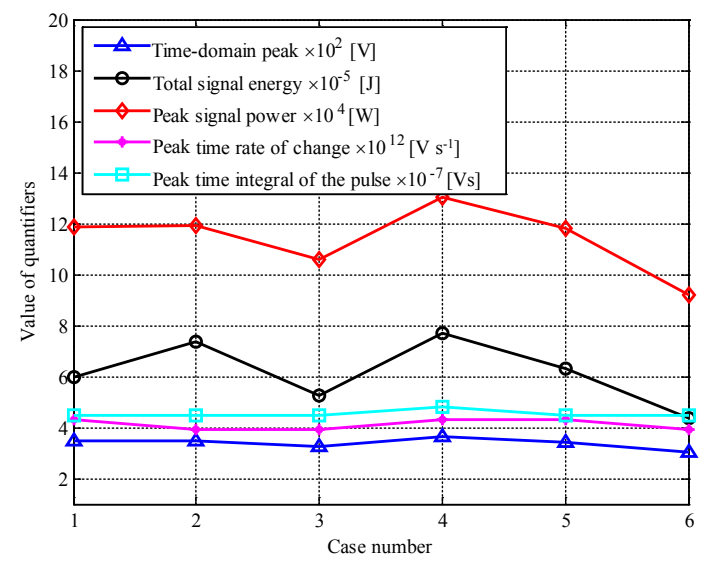

Figure 12. The maximum values of the five quantifiers, when changing the lengths of power lines.

pact of UWB transient on the targeted load, regarding some quantifiers.

In light of the above, we should notice that, the lengths of power lines have significant effect on the overall trends of the five quantifiers while other configurations in the network remain unchanged. For certain power lines, e.g., $L_{0}$ or $L_{3}$, which is along the propagation path here, the variation of 
length may result in obvious changes in quantifiers' behavior (i.e., total signal energy and peak signal power). Therefore, when deploying a power-line network, it is crucial to take the lengths of power lines into account, especially for some critical power lines.

\section{Conclusions}

In this paper, we study the impact of IEMI on a targeted load, which is distributed in a specific multi-junction power-line network. Based on the modified BLT method, the investigation is performed in terms of five quantifiers, i.e., timedomain peak, total signal energy, peak signal power, peak time rate of change, and peak time integral of the pulse. This is done from the following aspects: the attribute of the loads (i.e., impedance), the rise time and pulse width of the UWB transient, and the lengths of power lines. The conclusions are drawn as follows:

1. In terms of the five common distinct evaluation quantifiers, a UWB source has a significant impact on a dominantly inductive or capacitive load. Thus, a targeted load can be made less vulnerable by increasing its resistive attribute and at the same time decreasing its amplitude $\left(\left|Z_{3}\right|\right)$.

2. For the UWB pulse, the $\beta / \alpha$ ratio plays an important role in affecting the quantifiers' behavior. To be more precise, when the ratio increases from 2 to 500 , the maximum values of some of the five quantifiers have a large growth.

3. It is crucial to take the lengths of power lines into account, since the length variation of critical power lines can result in significant changes of some quantifiers' behavior. It is also worth noting that, when the load is inductive (or capacitive), the quantifiers will suffer more at some length of power line, while they may not at other lengths.

4. It is important to investigate if the targeted load is susceptible more to one of five quantifiers than others.

Data availability. The data is available on request and the readers can contact the author via email (libing@kth.se).

Competing interests. The authors declare that they have no conflict of interest.

Edited by: Frank Gronwald

Reviewed by: Dave Giri and three anonymous referees

\section{References}

Anatory, J., Theethayi, N., and Thottappillil, R.: Power-line communication channel model for interconnected networks - Part I: Two-conductor system, IEEE Trans. Power Deliv., 24, 118-123, 2009.

Baum, C. E.: Generalization of the BLT equation, Interaction Note, 511, 1-136, 1995.

Baum, C. E., Liu, T. K., and Tesche, F. M.: On the analysis of general multiconductor transmission-line networks, Interaction Note, 350, 467-547, 1978.

Bayram, Y., Volakis, J. L., Myoung, S. K., Doo, S. J., and Roblin, P.: High-power EMI on RF amplifier and digital modulation schemes, IEEE T. Electromag. Compatibil., 50, 849-860, 2008.

Beek, S. and Leferink, F.: Robustness of a TETRA base station receiver against intentional EMI, IEEE T. Electromag. Compatibil., 57, 461-469, 2015.

Camp, M. and Garbe, H.: Parameter estimation of double exponential pulses (EMP, UWB) with least squares and Nelder Mead algorithm, IEEE T. Electromag. Compatibil., 46, 675-678, 2004.

Camp, M., Gerth, H., Garbe, H., and Haase, H.: Predicting the breakdown behavior of microcontrollers under EMP/UWB impact using a statistical analysis, IEEE T. Electromag. Compatibil., 46, 368-379, 2004.

Carlsson, J., Karlsson, T., and Undén, G.: EMEC-an EM simulator based on topology, IEEE T. Electromag. Compatibil., 46, 353$358,2004$.

Giri, D. and Tesche, F.: Classification of intentional electromagnetic environments (IEME), IEEE T. Electromag. Compatibil., 46, 322-328, 2004.

Hoad, R., Carter, N. J., Herke, D., and Watkins, S. P.: Trends in EM susceptibility of IT equipment, IEEE T. Electromag. Compatibil., 46, 390-395, 2004.

Li, B. and Månsson, D.: Frequency Response Analysis of IEMI in Different Types of Electrical Networks, in: Asia Electromagnetics Symposium (ASIAEM), 3-8 August 2015, Jeju, Republic of Korea, 2015.

Li, B. and Månsson, D.: Impact evaluation of conducted UWB transients on terminal loads in a network, in: European Electromagnetics Symposium (EUROEM), 11-15 July 2016, London, UK, 2016.

Li, B. and Månsson, D.: Effect of Periodicity in Frequency Responses of Networks from Conducted EMI, IEEE T. Electromag. Compatibil., https://doi.org/10.1109/TEMC.2017.2689924, in press, 2017.

Li, B., Månsson, D., and Yang, G.: An Efficient Method for Solving Frequency Responses of Power-Line Networks, Prog. Electromag. Res. B, 62, 303-317, 2015.

Månsson, D.: Intentional electromagnetic interference (IEMI): Susceptibility investigations and classification of civilian systems and equipment, PhD thesis, Uppsala University, Uppsala, 2008.

Månsson, D., Nilsson, T., Thottappillil, R., and Backstrom, M.: Propagation of UWB transients in low-voltage installation power cables, IEEE T. Electromag. Compatibil., 49, 585-592, 2007.

Månsson, D., Thottappillil, R., and Bäckström, M.: Propagation of UWB transients in low-voltage power installation networks, IEEE T. Electromag. Compatibil., 50, 619-629, 2008a.

Månsson, D., Thottappillil, R., Backstrom, M., and Lundén, O.: Vulnerability of European rail traffic management system to radi- 
ated intentional EMI, IEEE T. Electromag. Compatibil., 50, 101109, 2008b.

Månsson, D., Thottappillil, R., and Bäckström, M.: Methodology for classifying facilities with respect to intentional EMI, IEEE T. Electromag. Compatibil., 51, 46-52, 2009.

Mao, C. and Zhou, H.: Novel parameter estimation of double exponential pulse (EMP, UWB) by statistical means, IEEE T. Electromag. Compatibil., 50, 97-100, 2008.

Meng, H., Guan, Y. L., and Chen, S.: Modeling and analysis of noise effects on broadband power-line communications, IEEE T. Power Deliv., 20, 630-637, 2005.

Rachidi, F. and Tkachenko, S.: Electromagnetic field interaction with transmission lines: from classical theory to HF radiation effects, in: vol. 5, WIT Press, Southampton, Boston, 2008.

Radasky, W. A., Baum, C. E., and Wik, M. W.: Introduction to the special issue on high-power electromagnetics (HPEM) and intentional electromagnetic interference (IEMI), IEEE T. Electromag. Compatibil., 46, 314-321, 2004.
Sabath, F. and Mokole, E. L.: Ultra-wideband Short-pulse Electromagnetics 10, Springer, New York, 2014.

Shin, J., Lee, J., and Jeong, J.: Channel modeling for indoor broadband power-line communications networks with arbitrary topologies by taking adjacent nodes into account, IEEE T. Power Deliv., 26, 1432-1439, 2011.

Taylor, C. D. and Giri, D.: High-power microwave systems and effects, Taylor \& Francis, Washington, D.C., 1994.

Tesche, F. M.: Development and use of the BLT equation in the time domain as applied to a coaxial cable, IEEE T. Electromag. Compatibil., 49, 3-11, 2007.

Vallbe, B., Balcells, J., Bogonez-Franco, P., Mata, J., and Gago, $\mathrm{X}$.: Immunity of power line communications (PLC) in disturbed networks, in: 2011 IEEE International Symposium on Industrial Electronics, 27-30 June 2011, Gdansk, Poland, 1621-1625, 2011.

Weber, T. and ter Haseborg, J. L.: Measurement techniques for conducted HPEM signals, IEEE T. Electromag. Compatibil., 46, 431-438, 2004. 